\title{
Photographic surface photometry of the Southern Milky Way
}

\section{High-resolution surface photometries at $217 \mathrm{~nm}$ and $280 \mathrm{~nm}$}

\author{
L. Schmidtobreick, W. Schlosser, P. Koczet, S. Wiemann, and M. Jütte \\ Astronomisches Institut der Ruhr-Universität Bochum, D-44780 Bochum, Germany
}

Received January 15, 1997; accepted March 9, 1998

\begin{abstract}
We present the first surface photometries of the Southern Milky Way $\left(100^{\circ} \leq l \geq 270^{\circ} ;-40^{\circ} \leq b \leq 40^{\circ}\right)$ at $217 \mathrm{~nm}$ and $280 \mathrm{~nm}$. The resolution is $0.25 \times 0.25$. They are based on photographs obtained with the shuttle-borne GAUSS-Camera as part of the German Spacelab Mission D-2. These photometries are the extensions of the earthbound $U, B, V, R$ photometries (Kimeswenger et al. 1993 = Paper VII; Hoffmann et al. $1997=$ Paper VIII) to the ultraviolet. The data processing was modified to adapt it to the new data. Both photometries are strongly affected by interstellar extinction especially of local dark clouds. Hence, they provide a comprehensive overview of scattering caused light emission in our Galaxy.
\end{abstract}

Key words: Galaxy (the): structure — Galaxy (the): open clusters and associations - techniques: photometry interstellar medium: dust — interstellar medium: reflection nebulae - ultraviolet: general

\section{Introduction}

A large-scale photographic survey of the southern Milky Way in the classic Johnson passbands $U, B, V$ and $R$ was begun in 1971 by obtaining images at the site of the European Southern Observatory (ESO) at La Silla/Chile (Schlosser 1971). This program was then extended to the ultraviolet in 1993, when the GAUSS camera was part of the German Spacelab Mission D-2, flewn on the space shuttle Columbia (Schlosser 1993).

Numerous surface photometries of the Milky Way have been published previously, the major earlier ones being those of Pannekoek (1933) and Pannekoek \& Koelbloed (1949). Elsässer \& Haug (1960) have been the first to

Send offprint requests to: L. Schmidtobreick email: linda@astro.ruhr-uni-bochum.de present photoelectric measurements of the Milky Way with a reasonable resolution in well defined passbands. Other large scale surface photometries since then are those of Smith et al. (1970); Pfleiderer \& Mayer (1971); Classen (1971); Weinberg (1981); Toller (1989) and the Bochum ones (Paper VII and Paper VIII). However, all of these photometries are restricted to spectral domains close to the visual.

In the ultraviolet, surface photometries have been presented only for smaller areas like some reflection nebulae (Gordon et al. 1994; Witt et al. 1992) or cirrus clouds (Haikala et al. 1995). In the $140 \mathrm{~nm}-180 \mathrm{~nm}$ passband, the FAUST FUV telescope has measured about 5000 sources spread over the sky (Bowyer et al. 1993) and some efforts have been made to measure the ultraviolet sky brightness or background radiation (Joubert et al. 1983; Henry 1991; Bowyer 1991; Henry \& Murthy 1993). More recent analysis concerning the dust-scattering and the diffuse radiation in the FUV has been conducted by Sasseen \& Deharveng (1996) and Witt et al. (1997) (and references therein).

We here present surface photometries in the ultraviolet on larger scales. In Sects. 2 and 3 we give a short overview on the instrumentation and the data used for the photometries. The reduction is described in detail in Sect. 4, and in Sect. 5 we present the resulting maps yielding a comprehensive picture of the Southern Milky Way at $217 \mathrm{~nm}$ and $280 \mathrm{~nm}$ (see also Schmidtobreick 1997 and Wiemann 1997).

\section{The camera}

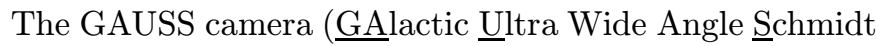
System) is an improvement of the Bochum Super Wide Angle Camera, which has been successfully used for the former, earthbound $U, B, V, R$ photometries (Paper VII and Paper VIII). It has been especially designed for the 


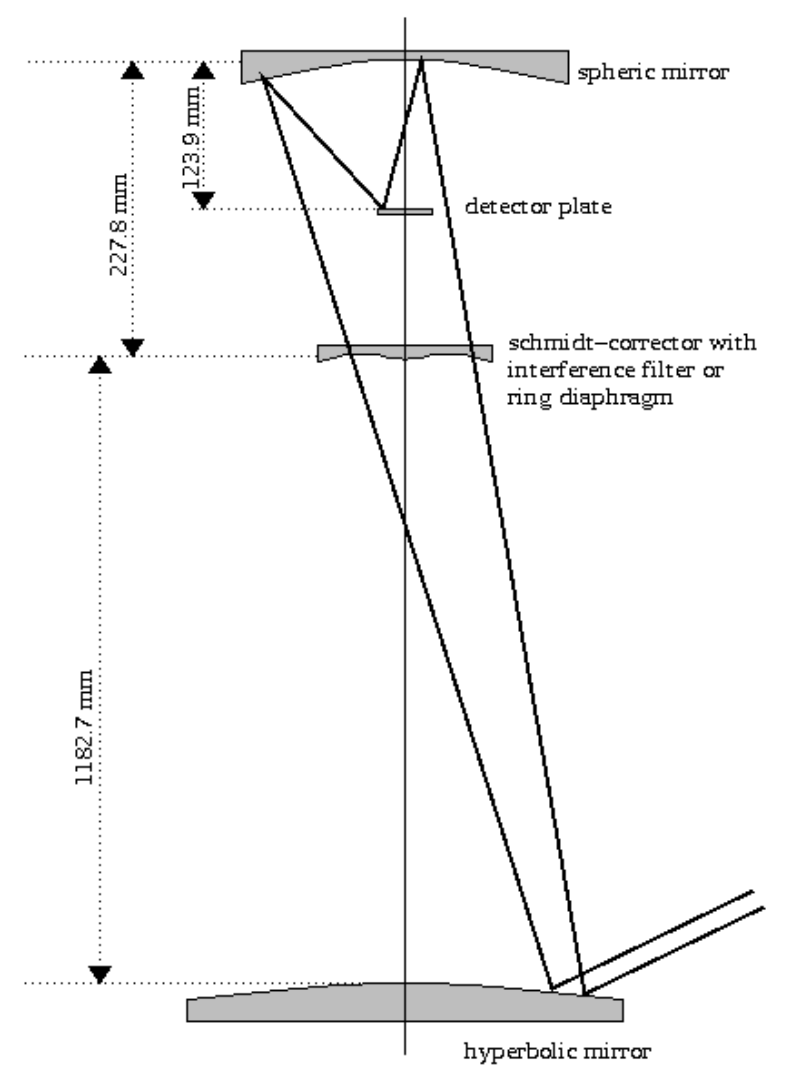

Fig. 1. The geometric concept of the GAUSS camera

investigation of surface brightnesses. This is achieved by the combination of the short focal length $f_{\text {center }}=11 \mathrm{~mm}$ with the high focal ratio $f / 2$. As main progress, the former spherical, concave mirror has been changed for a hyperbolic, convex one, and the imaging component is now a Schmidt system. This combination results in a flat focal plane without astigmatism. The mirror surface is made of aluminum with a protective covering of LiF. In Fig. 1, a sketch of the main components of the camera is given. A ring diaphragm is employed for broad band photometry, and for the colour images. Interference filters centered on $122 \mathrm{~nm}, 175 \mathrm{~nm}, 217 \mathrm{~nm}, 280 \mathrm{~nm}$ and $357 \mathrm{~nm}$ are combined with the Schmidt correctors and separate different passbands in the ultraviolet spectral range. All these nonreflective components are made of $\mathrm{MgF}_{2}$ or $\mathrm{SiO}_{2}$ depending on the wavelength. Figure 2 shows the transmission curves of the five filters, the sensitivity of the detector can be regarded as constant. Two detectors have been chosen for the UV-images: Kodak SO647 and Kodak 101-01, the latter one being more sensitive by a factor of four. Additionally, a couple of commercial negative and reversal colour films have been exposed. The idea and function of the camera and its linear mounting has been described in detail by Schlosser (1993) and by Schlosser \& Koczet (1995).
Table 1. Filter, detector, center coordinates, hour, and exposure-time of the selected photographs

\begin{tabular}{|c|c|c|c|c|c|}
\hline \multicolumn{6}{|c|}{$217 \mathrm{~nm}$} \\
\hline No & Detector & $l_{0}$ & $b_{0}$ & $\begin{array}{l}\text { MET } \\
\text { (d/h:min) }\end{array}$ & Exp--Time \\
\hline 006 & 101-01 & 314.5 & 39.9 & $0 / 17: 22$ & $17 \mathrm{~min}$ \\
\hline 049 & SO647 & 24.5 & 26.1 & $\begin{array}{l}\text { 4/19:32 } \\
4 / 21: 02 \\
4 / 22: 33\end{array}$ & $51 \mathrm{~min}$ \\
\hline 087 & SO647 & $316^{\circ} 9$ & $51^{\circ} .5$ & $\begin{array}{l}\text { 7/21:12 } \\
8 / 06: 09\end{array}$ & $39 \min$ \\
\hline 109 & SO647 & $280^{\circ} .2$ & $37^{\circ} .1$ & $\begin{array}{l}9 / 10: 42 \\
9 / 12: 12\end{array}$ & $34 \mathrm{~min}$ \\
\hline \multicolumn{6}{|c|}{$280 \mathrm{~nm}$} \\
\hline No & Detector & $l_{0}$ & $b_{0}$ & $\begin{array}{l}\text { MET } \\
\text { (d/h:min) }\end{array}$ & Exp--Time \\
\hline 022 & SO647 & 24.5 & 26.1 & $\begin{array}{l}1 / 22: 14 \\
1 / 23: 45 \\
2 / 01: 15\end{array}$ & $50 \mathrm{~min}$ \\
\hline 082 & SO647 & $280^{\circ} .2$ & $37^{\circ} .1$ & $\begin{array}{l}6 / 08: 53 \\
6 / 10: 24 \\
6 / 11: 54\end{array}$ & $51 \mathrm{~min}$ \\
\hline 086 & SO647 & 314.5 & 39.9 & $\begin{array}{l}7 / 16: 34 \\
7 / 18: 05 \\
7 / 19: 36\end{array}$ & $48 \mathrm{~min}$ \\
\hline
\end{tabular}

\section{Data}

Between April, 26th and May, 5th 1993, about 40 photographs of the Milky Way have been obtained with the GAUSS-Camera using the various filters described in Sect. 2. Each of them has a field of view of $140^{\circ}$. For this work, the best images in each filter have been chosen, four at $217 \mathrm{~nm}$ and three at $280 \mathrm{~nm}$. Their properties are given in Table 1. The images have been digitized with the PDS machine of the Astronomical Institute of the Westfälische Wilhelms-Universität, Münster, Germany, using a diaphragm of $20 \mu \mathrm{m} \times 20 \mu \mathrm{m}$ and a step size of $10 \mu \mathrm{m} \times 10 \mu \mathrm{m}$. This corresponds to a resolution of 0.05 in the sky.

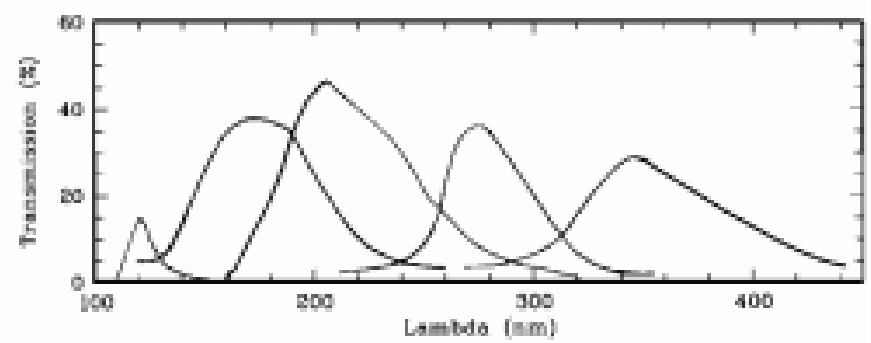

Fig. 2. The transmission of the GAUSS colour system 
Table 2. The coordinates of the tangential points, the parameters of distortion and offset, and the resulting errors are given for each image

\begin{tabular}{lllllllllll}
\hline No & $x_{\mathrm{C}} / \mu \mathrm{m}$ & $y_{\mathrm{C}} / \mu \mathrm{m}$ & $l_{\mathrm{C}}$ & $b_{\mathrm{C}}$ & $A$ & $B$ & $C$ & $\Delta \phi$ & $\sigma_{x} / \mu \mathrm{m}$ & $\sigma_{y} / \mu \mathrm{m}$ \\
\hline 006 & 16460.0 & 15803.0 & 314.212 & 39.930 & $7.325085 \mathrm{e}-6$ & $-6.455197 \mathrm{e}-5$ & 0.18483780 & 296.40737 & 5.185 & 4.726 \\
022 & 15192.0 & 15250.0 & 24.533 & 26.235 & $7.036284 \mathrm{e}-6$ & $-3.831281 \mathrm{e}-5$ & 0.18379405 & 236.27584 & 5.146 & 5.039 \\
049 & 15501.0 & 15634.0 & 316.878 & 51.501 & $7.163900 \mathrm{e}-6$ & $-6.185264 \mathrm{e}-5$ & 0.18453767 & 334.82393 & 3.455 & 3.221 \\
082 & 15616.0 & 15670.0 & 280.188 & 37.040 & $7.081593 \mathrm{e}-6$ & $-5.074773 \mathrm{e}-5$ & 0.18442495 & 178.19131 & 3.531 & 3.511 \\
086 & 16141.0 & 16005.0 & 314.222 & 39.939 & $7.025267 \mathrm{e}-6$ & $-4.831028 \mathrm{e}-5$ & 0.18436151 & 288.99714 & 4.493 & 3.507 \\
087 & 16137.0 & 15837.0 & 316.865 & 51.489 & $6.820321 \mathrm{e}-6$ & $-2.887512 \mathrm{e}-5$ & 0.18395928 & 327.13729 & 2.342 & 2.637 \\
109 & 15566.0 & 15567.0 & 280.216 & 37.080 & $7.128245 \mathrm{e}-6$ & $-5.490747 \mathrm{e}-5$ & 0.18448409 & 179.20094 & 2.957 & 3.208 \\
\hline
\end{tabular}

\section{Reductions}

\subsection{Geometric rectification}

Due to the precise manufacturing of the GAUSS camera, its distortion can be assumed as radially symmetric with respect to the optical axis of the system. Hence, the coordinates to describe this distortion have been chosen as spherical ones: the radial distance $r_{\mathrm{P}}$ of an image point from the center of a plane plate and the rotation angle $\phi_{\mathrm{P}}$. The catalogued galactic coordinates of about 120 stars on each image have been converted into celestial spherical coordinates: the radial distance $r_{\mathrm{S}}$ of the stars from the assumed tangential point in the sky and the rotation angle $\phi_{\mathrm{S}}$ starting from the meridian of the tangential point. An iteration around the center point of the plate and the estimated corresponding tangential point in the sky has been performed. For each step the spherical celestial coordinates have been compared with the spherical plate coordinates, a polynomial has been fitted to the data, and the deviation has been computed. This deviation has been minimized by the iteration resulting in the correct coordinates $\left(x_{\mathrm{C}}, y_{\mathrm{C}}, l_{\mathrm{C}}, b_{\mathrm{C}}\right)$ of the tangential point, and in the polynomials

$r_{\mathrm{P}}=A r_{\mathrm{S}}^{3}+B r_{\mathrm{S}}^{2}+C r_{\mathrm{S}}$

$\phi_{\mathrm{P}}=\phi_{\mathrm{S}}+\Delta \phi$

describing the distortion and the angular offset of the camera. The parameters for the selected images are given in Table 2. The mean error of this rectification is about $\pm 4 \mu \mathrm{m}$ or 0.02 in the sky.

\subsection{Photometric calibration}

The former Bochum surface photometries (Paper VII; Paper VIII) have been calibrated by the mean of gradation curves that have been exposed using ESO's wedge spectrograph ETA. Additionally, during the photographic exposures absolute calibration data and information on airglow, scattered light and extinction have been measured photoelectrically.

Since none of these measurements has been done during the spacebound observation, the gradation curves had to be reconstructed from the images themselves. This requires measuring the star densities to compare them with their catalogued fluxes. As reference, the catalogues of Jamar et al. (1976) and of Thompson et al. (1978) have been taken, both resulting from the measurements of the satellite TD 1. The stars have been identified on the images, the maximum of their density has been determined, and for all recovered stars (about 1500 on each image) these maxima have been set in relation to the catalogued flux. The transformation from the measured densities to relative intensities were done by using the characteristic curve after Moffat (1969)

$D=\frac{\gamma}{n} \lg \left(1+\left(\frac{I}{I_{0}}\right)^{n}\right)+D_{0}$

where $D$ is the measured density, and $D_{0}$ the density of the chemical fog of the photograph. $\gamma, n$ and $I_{0}$ are free parameters that have been derived by fitting the curve to the data. The typical mean error of this calibration is about $2 \%$.

Absolute calibration was obtained by integrating the relative photographic intensities of each identified star, subtracting its individual sky background, and comparing the resulting aperture star photometries $F_{\mathrm{AP}}$ with the catalogued fluxes $F_{*}$. This yields a linear relation with a typical mean error of about $3 \%$.

To obtain the surface photometries, the calibrated images have been divided by $(5.37 \pm 0.1) 10^{-7} \mathrm{sr}$ which is the mean area of one pixel. The parameters $D_{0}, \gamma, n$ and $I_{0}$ of the relative calibration, the ratio $F_{\mathrm{AP}} / F_{*}$ describing the absolute calibration, and the resulting mean error are given in Table 3 for each image.

Table 3. The calibration parameters are given for each image

\begin{tabular}{lllllll}
\hline No. & $D_{0}$ & $\gamma$ & $n$ & $I_{0}$ & $F_{\text {AP }} / F_{*}$ & $\sigma$ \\
\hline 006 & 510 & 1268.25 & 0.451037 & 32.1979 & 2.8206 & $2.9 \%$ \\
022 & 305 & 1050.83 & 0.64040 & 11.0106 & 4.8661 & $3.2 \%$ \\
049 & 365 & 1200.85 & 0.569398 & 37.9737 & 4.5105 & $3.1 \%$ \\
082 & 405 & 890.09 & 0.772199 & 2.90174 & 3.9290 & $3.3 \%$ \\
086 & 395 & 778.58 & 0.6206 & 3.56952 & 4.9211 & $3.1 \%$ \\
087 & 310 & 872.64 & 0.666108 & 10.8252 & 4.7543 & $3.5 \%$ \\
109 & 410 & 1171.67 & 0.483996 & 68.6545 & 3.5806 & $3.7 \%$ \\
\hline
\end{tabular}




\subsection{Elimination of foreground stars}

The elimination of disturbing, individual foreground stars has been done in two steps. First, all stars brighter than $310^{-14} \mathrm{~W} / \mathrm{m}^{2} \mathrm{~nm}$ have been removed from the images. These stars have a characteristic intensity profile that has been used for their identification. Beginning in the center of each star, the average radial differences have been computed and compared to the inner error. As soon as the difference became smaller than the mean error, the hereto belonging circle was defined as the "edge" of the star and the intensity inside this circle was replaced by the average of the individual background.

The fainter stars which do not have this characteristic profile do only cover an area of maximally four pixel and could be removed by a filter similar to the one described in Paper VII. Herewith, all stars brighter than $2.510^{-15} \mathrm{~W} / \mathrm{m}^{2} \mathrm{~nm}$ have finally been eliminated from the images.

\subsection{Zodiacal light and Shuttle Glow}

To correct for zodiacal light, we used the values published by Tennyson et al. (1988). Since the zodiacal light at $217 \mathrm{~nm}$ and $280 \mathrm{~nm}$ is distributed quite homogeneously (Murthy et al. 1990), and since its intensity is also very small, it is sufficient to substract an average value from each image. This value has been determined to $(0.9 \pm 0.3) 10^{-11} \frac{W}{\mathrm{~m}^{2} \mathrm{sr} \mathrm{nm}}$ at $217 \mathrm{~nm}$ and to $(5.7 \pm 2) 10^{-11} \frac{W}{\mathrm{~m}^{2} \mathrm{srnm}}$ at $280 \mathrm{~nm}$.

Much stronger is the additional light emission that is produced by the shuttle while interacting with the upper atmosphere. Many of the images are influenced by these shuttle glow effects (see Jütte 1996 for a thorough investigation of these phenomena). The classical Shuttle Glow is generally considered to be relatively strong in the red and near-infrared passbands but negligible in the UV. However, we have detected quite intense light phenomena at shorter wavelengths that seem to be related to the shuttle: large, bright clouds surrounding the shuttle and even the camera itself. They seem to be of variable origin and change on timescales of seconds to hours. To eliminate these clouds from the images, they have been fitted with twodimensional polynomials of fourth order. These fits reproduced the shape of the clouds in a reasonable way, however, the zero point of the sky background could not be derived by this method. Therefore it has been estimated from the darkest parts of the images, which have been regarded as unaffected by the glow. The error of this estimation is very high with about $20 \%$ of the mean Milky Way brightness. However, there is no other possibility to derive the correct sky background. We consider these glow phenomena to be a major obstacle to all photometries obtained at Low Earth Orbits.

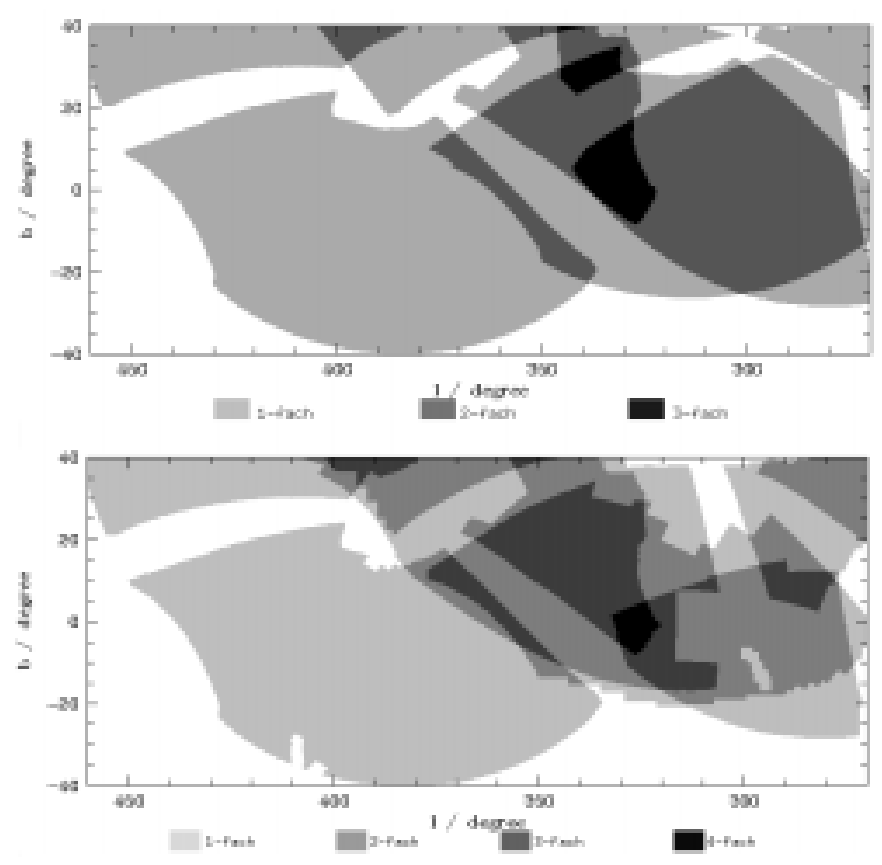

Fig. 3. The number of images that have been averaged for each point of the final maps (above: $280 \mathrm{~nm}$; below: $217 \mathrm{~nm}$ )

\subsection{Transformation and averaging}

The derivation of the geometric parameters (Table 2) allows the transformation of the images into maps of the Milky Way in cartesian coordinates $l, b$. The stepsize has been chosen as $0.25 \times 0.25$ for comparison with the $U, B, V, R$ photometries (Paper VII; Paper VIII). The individual maps have been added up to give a picture of the Milky Way by averaging the overlapping regions. Due to the small number of usable images, there are only a few regions where the sky coverage is equal or better than three images (see Fig. 3).

\section{The resulting photometries}

In Figs. 5 and 6 the final surface photometries are presented. Both maps cover the Milky Way between Cygnus and Vela $\left(100^{\circ} \leq l \geq 270^{\circ},-40^{\circ} \leq b \leq 40^{\circ}\right)$. Figure 4 shows the same maps as contour plots. The appearance of the Milky Way at $217 \mathrm{~nm}$ and $280 \mathrm{~nm}$ is dominated strongly by interstellar extinction, leading to high intensity gradients all over the galactic plane. Especially, the effect by local dark clouds is extremely strong. Together with young OB stars emitting high flux densities in the UV, they are responsible for various bright reflection nebulae and other light emissions that can be attributed to scattering phenomena.

Other bright sources on the photometries are near, young open clusters like NGC 3114, IC 2602, M6, M7, M 8, and M 20. Since these objects consist mainly of OB stars, the bright appearence of them is to be expected. 

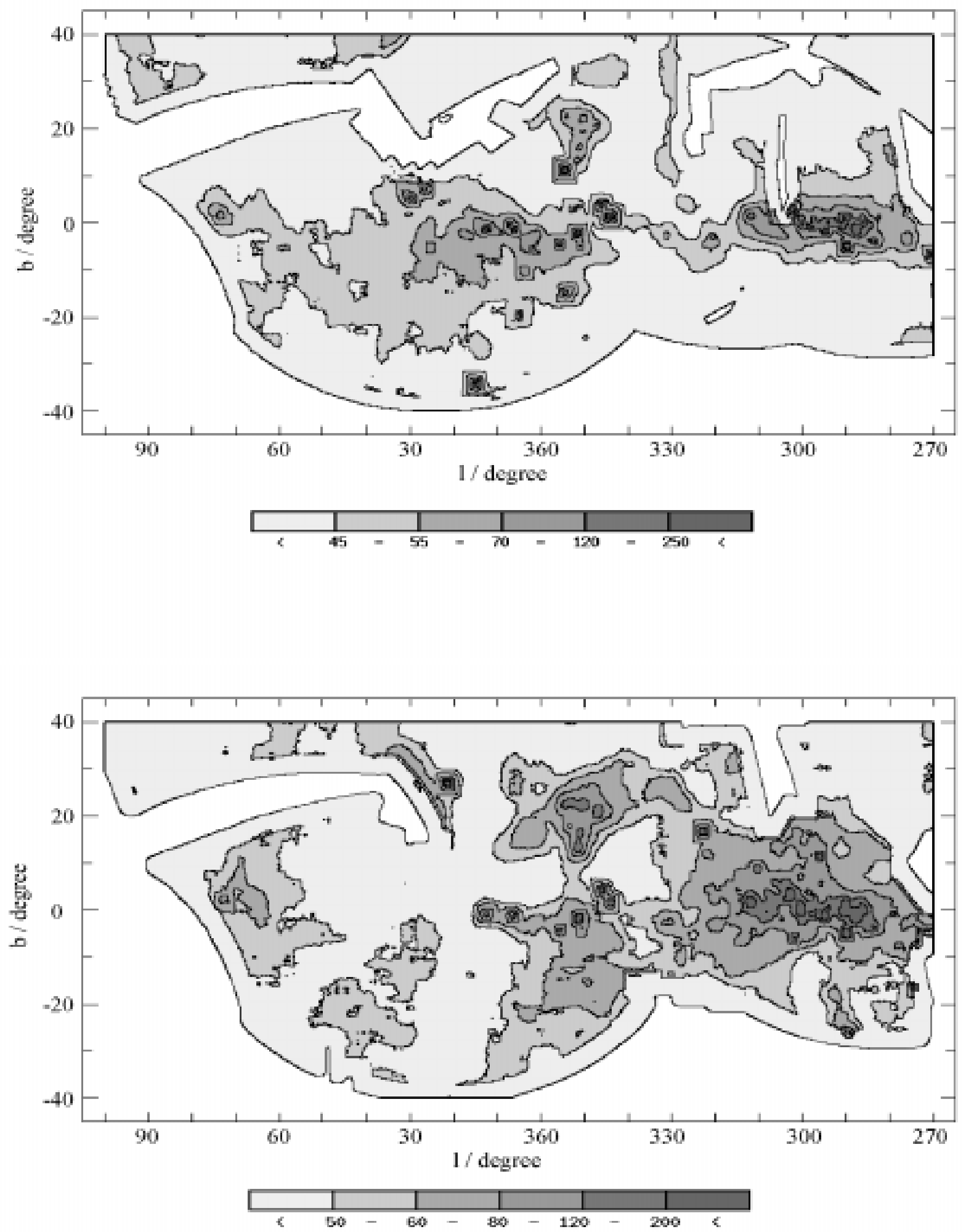

Fig. 4. Contour plots of the photometries of the Milky Way at central wavelengths of $280 \mathrm{~nm}$ (above) and $217 \mathrm{~nm}$ (below). The units for the given intensity steps are $10^{-11} \frac{W}{\mathrm{~m}^{2} \mathrm{~nm} \mathrm{sr}}$. For clarity, the areas between the contours are coloured as indicated in the greyscales 


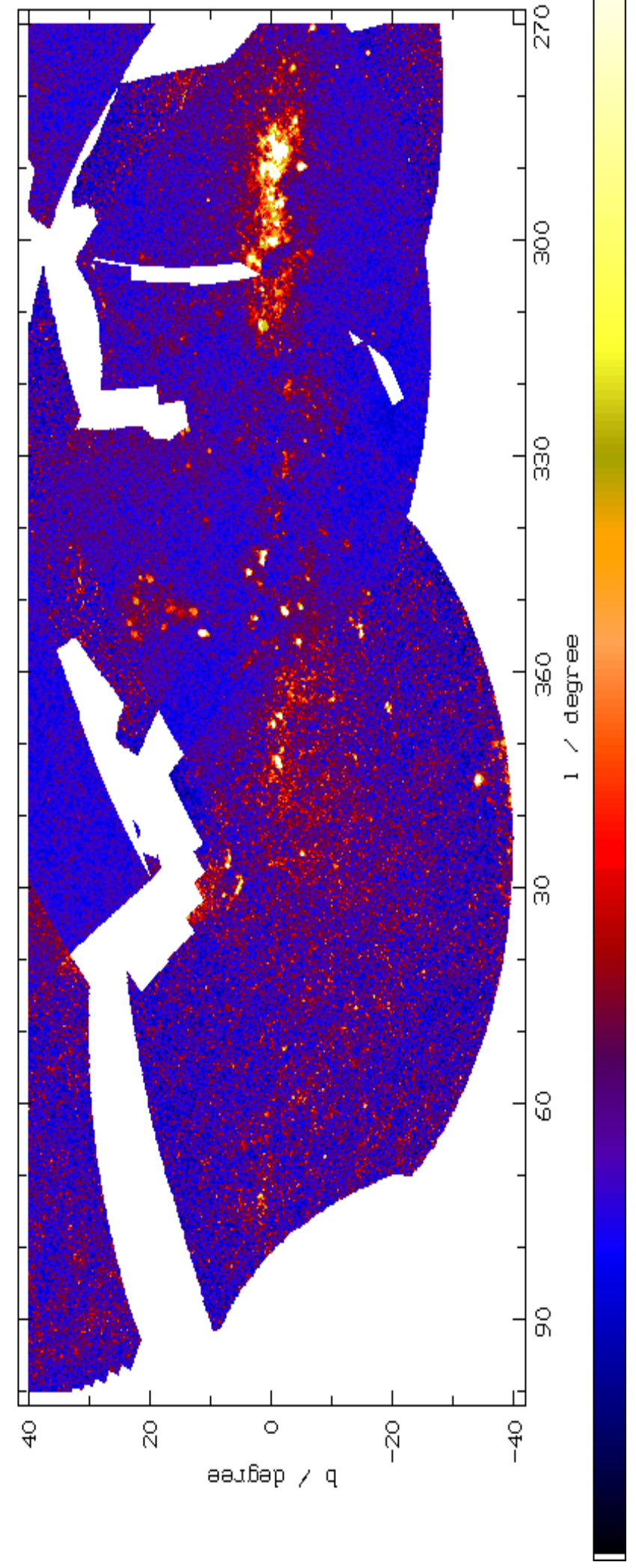

Fig. 5. The photometric map of the Southern Milky Way at a central wavelength of $280 \mathrm{~nm}$. The colour coded intensity range is (black) $\leq-50 \ldots 35010^{-11} \frac{W}{\mathrm{~m}^{2} \mathrm{nmsr}} \leq$ (white)

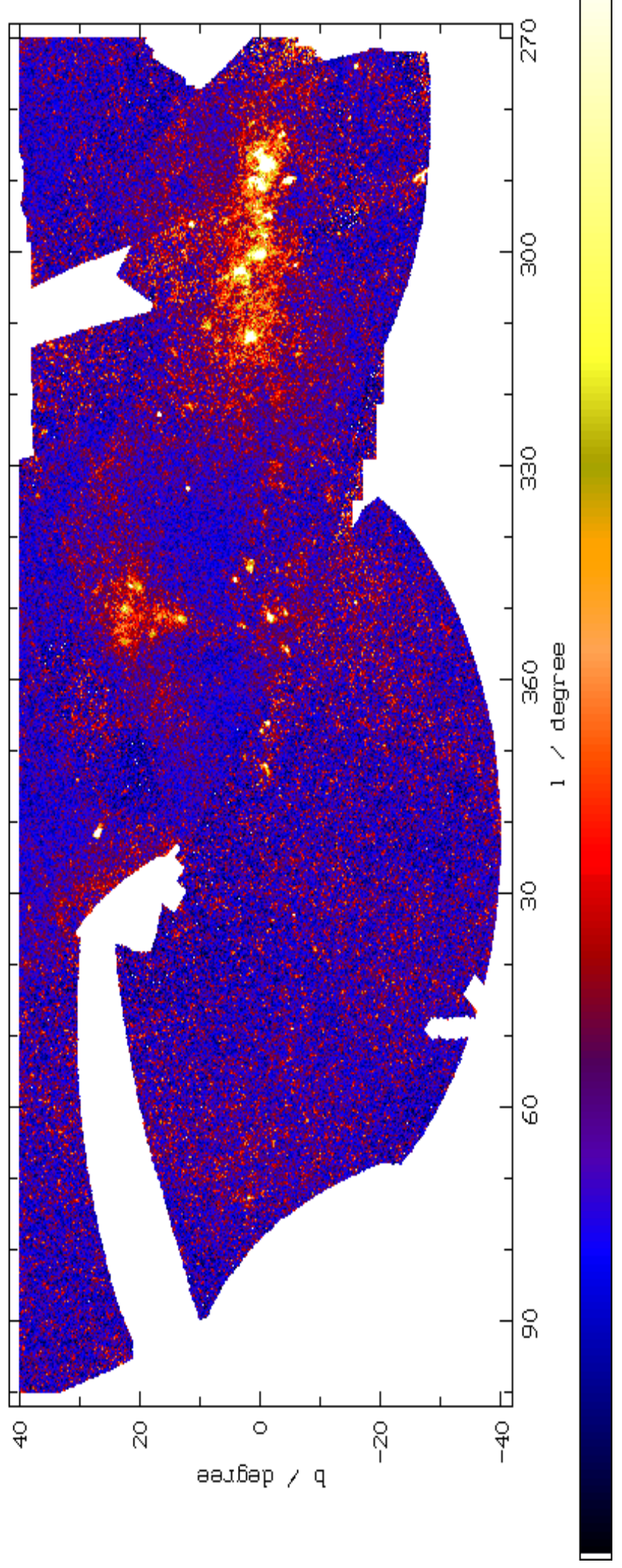

Fig. 6. The photometric map of the Southern Milky Way at a central wavelength of $217 \mathrm{~nm}$. The colour coded intensity range is (black) $\leq-50 \ldots 40010^{-11} \frac{W}{\mathrm{~m}^{2} \mathrm{~nm} \mathrm{sr}} \leq$ (white) 
The region around $\eta$ Car, where the tangential point of the Sgr-Car spiral arm is assumed, is one of the brightest areas in both photometries. This can be explained by two effects. First, in this direction the extinction is assumed to be very low (see e.g. Neckel \& Klare 1980). Even in the UV, one can see deep into our galaxy onto the inner spiral arm. Second, spiral arms are dominated by young, hot stellar objects. Hence, the integrated star light of many sources bright in the UV forms the high surface intensities in this direction.

The galactic center region is hardly visible at all. Here, the stellar population building the bulge is a relatively old one. Although there is no agreement on the exact age of the bulge, it is generally accepted, that it ought to be older than 5-8 Gyr (Holtzman et al. 1993; Bertelli et al. 1995 and others in there). The resulting low number of young, hot stars, is responsible for the low surface intensity in this region. Additionally, the effects of the large dark clouds of the Ophiuchus complex must not be neglected. The whole region around the galactic center is almost completely hidden by this complex.

Comparing the appearance of the Milky Way in the UV with the earthbound $U B V R$ photometries shows a straight line of order. Towards shorter wavelengths, the image of the Milky Way becomes more and more disrupted due to the increasing effects of interstellar extinction. The galactic center, which is the brightest part in $R$, becomes fainter, whereas the surface brightness of the Carinae region increases and begins to exceed the galactic center brightness in $B$.

\section{Summary}

Two surface photometries of the southern Milky Way have been established at central wavelengths $217 \mathrm{~nm}$ and $280 \mathrm{~nm}$. Except for some windows of low extinction in the interstellar dust, all the light phenomena on the maps are local and can be attributed to the integrated light of young hot stellar objects or to scattering phenomena resulting from the interaction of OB starlight with the interstellar grains.

The data will be soon accessible in digital form at the astronomical data center Centre de Données Stellaires (CDS) in Strasbourg under

\section{http://cdsweb.u-strasbg.fr/htbin/myqcat3?VII/199/}

At this address, all major surface photometries of the Milky Way shall be collected and made accessible to the public.

Acknowledgements. The authors are grateful to Prof. Dr. W.C. Seitter and her staff of the Astronomisches Institut der Westfälischen Wilhelms-Universität Münster for granting measuring time and technical support. We also thank the Deutsche Agentur für Raumfahrtangelegenheiten (DARA) for financial support.

\section{References}

Bertelli G., Bressan A., Chiosi C., Ng Y.K., Ortolani S., 1995, A\&A 301, 381

Bowyer S., 1991, ARA\&A 29, 59

Bowyer S., Sasseen T., Lampton M., Wu X., 1993, ApJ 415, 875

Classen Ch., 1971, PhD Thesis, University of Bonn

Elsässer H., Haug U., 1960, ZfA 50, 121

Gordon K.D., Witt A.N., Carruthers G.R., Christensen S.A., Dohne B.C., 1994, ApJ 432, 641

Haikala L.K., Mattila K., Bowyer S., Sassen Th.P., Lampton M., Knude J., 1995, ApJ 443, L33

Henry R.C., 1991, ARA\&A 29, 89

Henry R.C., Murthy J., 1993, ApJ 418, L17

Hoffmann B., Tappert C., Schlosser W., Schmidt-Kaler Th., Kimeswenger S., Seidensticker K., Schmidtobreick L., Hovest W., 1997, A\&AS (in press) (Paper VIII)

Holtzman J.A., Light R.M., Baum W.A., Worthey G., Faber S.M., Hunter D.A., O'Neil E.J. Jr., Kreidl T.J., Groth E.J., Westphal J.A., 1993, AJ 106, 1826

Jamar C., Macau-Hercot D., Monfils A., Thompson G.I., Houziaux L., Wilson R., 1976, Ultraviolet bright-star spectrophotometric catalogue

Joubert M., Deharveng J.M., Cruvellier P., Masnou J.L., Lequeux J., 1983, A\&A 128, 114

Jütte M., 1996, Diploma Thesis, Ruhr-Universität Bochum

Kimeswenger S., Hoffmann B., Schlosser W., Schmidt-Kaler Th., 1993, A\&AS 97, 517 (Paper VII)

Moffat A.F.J., 1969, A\&A 3, 455

Murthy J., Henry R.C., Feldman P.D., Tennyson P.D., 1990, A\&A 231, 187

Neckel T., Klare G., 1980, A\&AS 42, 251

Pannekoek A., 1933, Publication of the Astronomical Institute of the University of Amsterdam, 3

Pannekoek A., Koelbloed D., 1949, Publication of the Astronomical Institute of the University of Amsterdam, 9, p. 28

Pfeiderer J., Mayer U., 1971, AJ 76, 691

Sasseen T.P., Deharveng J.-M., 1996, ApJ 469, 691

Schlosser W., 1972, Habilitationsschrift, Ruhr-Universität Bochum

Schlosser W., 1993, in "Research in Space", Sahm, Keller, Schiewe (ed.), WPR Köln

Schlosser W., Koczet P., 1995, Proc. of the Norderney Symp., WPR Köln

Schmidtobreick L., 1997, PhD Thesis, Ruhr-Universität Bochum

Smith L.L., Roach F.E., Owen R.W., 1970, Batelle Institute Report, BNWL-1419-UC-2

Tennyson P.D., Henry R.C., Feldman P.D., Hartig G.F., 1988, ApJ 330, 435

Thompson G.I., Nandy K., Jamar C., Monfils A., Houziaux L., Carnochan, D.J., Wilson R., 1978, Catalogue of stellar ultraviolet fluxes, Science Research Council, p. 23

Toller G.N., 1989, IAU Symp. 139, 21

Weinberg J.L., 1981, Sky and Telescope 61, 114

Wiemann S., 1997, Diploma Thesis, Ruhr-Universität Bochum

Witt A.N., Friedmann B.C., Sasseen T.P., 1997, ApJ 481, 809

Witt A.N., Petersohn J.K., Bohlin R.C., O'Connell R.W., Roberts M.S., Smith, A.M., Stecher T.P., 1992, ApJ 395, L5 